\title{
Keep calm and carry on with small tweaks: teamwork in the pandemic
}

\author{
Weijia Li \\ University of Rochester, New York, USA
}

Keywords: teamwork; division of labor; use of tools; local innovations; Covid-19.

\section{The challenge}

In March 2020, we pivoted to working remotely, as the pandemic unfolded itself. As one of the offices nested in the teaching and learning centre in a private research university in the United States, our study skills consulting team at the time was made up of four consultants: three full-time graduate students (including me) and a full-time assistant director as the team leader. We were juggling multiple commitments respectively before the pandemic. However, when we were stationed at home, holding appointments with students via Zoom, the change revealed issues we previously seemed to handle well, one of which was communication. The timely casual chats had disappeared since we no longer saw each other at the office. For example, in a face-to-face environment, informing a colleague of project updates could take a few minutes. However, doing so via email could take much longer. Certainly, discussing over Zoom might hasten the process, but that would require additional time and effort to set up such a meeting. Consequently, we seemed to have become solely reliant on verbal communication, including emails and weekly Zoom meetings. Operating remotely largely diminished nonverbal, and put more strain on verbal, communication. Meanwhile, online communication, while viable, had influenced our sense of efficiency and progress.

\section{The response}

My doctoral coursework on learning theories and research offered me some tools to brainstorm possible strategies, particularly the notion of activity system (Greeno and 
Engeström, 2013). Because the unit of analysis for an activity system is more than for an individual person, it appears an appropriate lens for understanding teamwork. However, an activity system itself does not remain unchanged. On the contrary, there is often timebound development driven by 'tensions, disturbances and local innovations' (Cole and Engeström, 1993, p.8). The pandemic disrupted the ways we used to work together and forced us to re-evaluate and respond. Therefore, we developed new practices as our way to respond and adapt to the disruption.

In order to strengthen transparency and timeliness in communication, I initially had the idea that we could use a virtual bulletin board to document our work and to-do items. With that, we were able to communicate throughout the week without emailing back and forth to ensure that everyone was on the same page. In addition, we could better prepare for our weekly meetings, knowing what we would be discussing. After I described how this could work, a colleague started a Google Doc to share among us. It also made it easier for us to keep track of what we had done. As a result, we began to realise and appreciate the benefits of documenting our work.

Second, I suggested a more specific division of labour (Marken, 2008) among team members to optimise efficiency and further reduce confusion in communication. Prior to working from home, each consultant (i.e., student employees) held office hours at designated times on weekdays. Because our primary duty was conducting one-on-one appointments with students, most of time we operated individually and did some ad hoc work when needed. The latter might continue for a while and naturally go through more than one person. However, when we started working from home, we increasingly felt the need to know 'who's doing what'. For example, we delegated among ourselves which departments we each should reach out to for collaboration, which was also reflected on the shared Google Doc. We were able to dedicate most of our time and effort to creating outreach initiatives, reducing the need to manage the logistics.

Furthermore, while having a specific division of labour seemed to make us work even more independently, we in fact collaborated more. Since 2020, we have witnessed a growing number of requests for virtual workshops and presentations, some of which were recorded. When happening virtually, these events tended to add more pressure to the presenter(s). One observation is that when the audience has not much to focus on visually, other than the slides and the presenter(s), they seem to focus more on the listening. At 
least that happened to me. I listened more attentively; however, it was easy for me to lose interest when a presenter did not sound well-prepared. Realising that, I started writing scripts for workshops and presentations because I could easily look at the script while presenting without it being noticed on camera. For one thing, the actual delivery felt stressfree, given that we could edit and tweak what we wanted to say in advance. For another, the scripts became artefacts that could be compiled for later. Not only were we able to showcase ourselves professionally with quality work, but we were also able to create a pool of resources for ourselves.

\section{Recommendations}

Our lessons are still relevant in a post-pandemic world, as many institutions plan to return to in-person instruction later this year. Although our study skills consulting team remains a small unit, thinking about mediating artefacts, or the tools we use, as well as the division of labour has been helpful. Start with tools you have already been using. Most likely, you may not need to incorporate new tools but perhaps invent new ways to use them. Next, delegate tasks for a clearer division of labour among team members; this creates some structure and the sense of direction that teamwork usually relies on. The pandemic has changed our way of working but also provided food for thought, reflection, and improvement. While uncertainty remains, we will keep reflecting and applying what we have learned to our future work.

\section{References}

Cole, M. and Engeström, Y. (1993) 'A cultural-historical approach to distributed cognition', in Salomon, G. (ed.) Distributed cognition: psychological and educational considerations. Cambridge: Cambridge University Press, pp.1-46.

Greeno, J. G. and Engeström, Y. (2013) 'Learning in activity’, in Sawyer, R. K. (ed.) The Cambridge handbook of the learning sciences. Cambridge: Cambridge University Press, pp.79-96. 
Marken, J. A. (2008) 'An application of activity theory', Performance Improvement Quarterly, 19(2), pp.27-49. https://doi.org/10.1111/j.1937-8327.2006.tb00364.x.

\section{Author details}

Weijia Li is a doctoral student in Teaching and Curriculum at the Warner School of Education, University of Rochester. She is one of the co-authors of the book titled An A to W of Academic Literacy: Key Concepts and Practices for Graduate Students, published in March 2021 by the University of Michigan Press. 